Jpn. J. Infect. Dis., 74, 405-410, 2021

Original Article

\title{
How Frequently Do Ordinary Citizens Practice Hand Hygiene at Appropriate Moments during the COVID-19 Pandemic in Japan?
}

\author{
Masaki Machida ${ }^{1,2}$, Itaru Nakamura ${ }^{2}$, Reiko Saito ${ }^{3}$, Tomoki Nakaya ${ }^{4}$, Tomoya Hanibuchi ${ }^{4}$, Tomoko Takamiya ${ }^{1}$, \\ Yuko Odagiri ${ }^{1}$, Noritoshi Fukushima ${ }^{1}$, Hiroyuki Kikuchi ${ }^{1}$, Shiho Amagasa ${ }^{1}$, Takako Kojima ${ }^{5}$, \\ Hidehiro Watanabe ${ }^{2}$, and Shigeru Inoue ${ }^{1 *}$
}

\author{
${ }^{I}$ Department of Preventive Medicine and Public Health, and \\ ${ }^{5}$ Department of International Medical Communications, Tokyo Medical University, Tokyo; \\ ${ }^{2}$ Department of Infection Prevention and Control, Tokyo Medical University Hospital, Tokyo; \\ ${ }^{3}$ Division of International Health (Public Health), Graduate School of Medical and Dental Sciences, Niigata \\ University, Niigata; and ${ }^{4}$ Graduate School of Environmental Studies, Tohoku University, Miyagi, Japan
}

\begin{abstract}
SUMMARY: We aimed to clarify the status of hand hygiene practices among ordinary citizens during the COVID-19 pandemic in Japan, as well as the frequency of daily hand hygiene as an indicator of education and evaluation. This cross-sectional study was based on an internet survey completed by 2,149 participants (age range: $20-79$ years, men: $51.0 \%$, response rate: $89.5 \%$ ), selected from June 23 to 28,2020 . The participants responded regarding the frequency of implementing hand hygiene at 5 moments (after returning from a public place, after using the toilet, after touching something outside, before eating food, and after blowing the nose, coughing, or sneezing). Additionally, the participants responded to the number of daily hand hygiene events. The cutoff value of the total number of daily hand hygiene events to determine whether hand hygiene was performed at all 5 time points was determined using receiver operating characteristic analysis. The mean number of hand hygiene events was 10.2 times/day. The prevalence of implementing hand hygiene at each moment ranged from $30.2 \%$ to $76.4 \%$; only $21.1 \%$ of respondents practiced hand hygiene at all times. Both Youden Index and specificity were high when the cut-off value was 11 times/day. Therefore, the criterion of hand hygiene ( $\geq 11$ times/day) may be useful in education and evaluation.
\end{abstract}

\section{INTRODUCTION}

As of October 2020, the coronavirus disease 2019 (COVID-19) pandemic remained active. Personal protective measures are implemented by the public as a method to mitigate the epidemic of respiratory viruses such as COVID-19, especially before a well-matched vaccine becomes widely available (1). Transmission of COVID-19 occurs primarily via respiratory droplets from face-to-face contact and, to a lesser degree, via contaminated surfaces $(2,3)$. To reduce transmission via respiratory droplets, certain researchers and health authorities have pointed out that wearing face masks in public spaces can be effective $(4,5)$. One of the personal

Received July 20, 2020. Accepted December 28, 2020.

J-STAGE Advance Publication January 29, 2021.

DOI: 10.7883/yoken.JJID.2020.631

*Corresponding author: Mailing address: Department of Preventive Medicine and Public Health, Tokyo Medical University, 6-1-1 Shinjuku, Shinjuku-ku, Tokyo 160-8402, Japan. Tel: +81-3-3351-6141, Fax: +81-3-3353-0162, E-mail: inoue@tokyo-med.ac.jp protective measures recommended by the World Health Organization (WHO) during the COVID-19 pandemic is hand hygiene (6). Contact transmission occurs when contaminated hands come into contact with the mucosa of the mouth, nose, or eyes; the virus can also be transferred from one surface to another by contaminated hands, which facilitates indirect contact transmission $(1,7)$. Consequently, hand hygiene is extremely important for preventing the spread of the virus.

Providing detailed instructions and precise "moments" for practicing hand hygiene is important for this specific measure. The WHO recommends that citizens regularly and thoroughly clean their hands using an alcohol-based hand rub or wash them with soap and water (6). On the other hand, the Japanese Ministry of Health, Labour and Welfare (MHLW) and the United States Centers for Disease Control and Prevention (CDC) recommend specific instances that require hand hygiene, such as before meals and after returning home $(8,9)$. Identifying specific moments for hand hygiene may be effective in increasing the number of hand hygiene events: the MHLW recommends 6 specific situations or moments, while the CDC recommends 12 situations $(8,9)$. This 
amount of information may be overwhelming for ordinary citizens, which could impede its dissemination. Needless to say, it is ideal for ordinary citizens to understand the moments when hand hygiene should be followed and practiced. However, ordinary citizens frequently have questions regarding how often hand hygiene should be practiced in daily life. With this in mind, we believe that it is possible to convey a more comprehensible and clearer educational message by identifying the number of hand hygiene practices and specific moments. Further, when evaluating the status of hand hygiene implementation among specific groups, such as employed workers and residents, it is necessary to know whether hand hygiene is being implemented when it should be. Nevertheless, inquiring about the status of implementation at each moment using questionnaires, etc., is a difficult task, given the large number of items, which can pose an obstacle in the evaluation of large groups. In contrast, the "number of hand hygiene events per day" is an indicator that is easy to determine using a questionnaire. Using the number of events as a criterion allows for a relatively simple assessment of the status of implementation. To identify the adequate number of hand hygiene practices, it is necessary to determine the approximate frequency at which hand hygiene is practiced by an individual during appropriate moments, as well as the total number of events. To the best of our knowledge, this is the first study of this kind.

Therefore, the purpose of this study was to elucidate the status of hand hygiene implementation among ordinary citizens in Japan during the COVID-19 pandemic and to clarify the number of hand hygiene practices per day as an indicator of awareness and assessment.

\section{MATERIALS AND METHODS}

Study sample and data collection: This crosssectional study utilized data from the 4 th wave of longitudinal research, which aimed to clarify the implementation of personal protective measures by ordinary citizens during the COVID-19 pandemic in Japan. In this longitudinal study, the implementation status of preventive measures by ordinary citizens during the COVID-19 pandemic was investigated approximately every 6 weeks. In the first wave survey of this longitudinal study on February 25, 2020, a total of 2,400 men and women aged 20-79 years (sampling by sex and 10-year age groups; 12 groups, $n=200$ in each group) who were living in seven prefectures (i.e., Tokyo, Kanagawa, Saitama, Chiba, Ibaraki, Tochigi, and Gunma), and who met the criteria to participate in this study were recruited from 8,156 registrants of a Japanese Internet research service company called MyVoice Communication, Inc. (Tokyo, Japan), which had approximately 1.12 million registered participants as of January 2020. Detailed sampling methods have been reported elsewhere $(10,11)$. The company reached out to these 2,400 potential respondents by e-mail to participate in the 4th wave of research on June 23, 2020. At the time, the daily number of reported COVID-19 cases in Japan was small (12); the Japanese government had already lifted the state of emergency on May 25
(13). The questionnaires were placed in a secured section of a website. Potential respondents received a specific link in their e-mail. Participation was voluntary; the participants responded to the questionnaire by accessing the specified link. The response deadline was June 28, 2020. There were a total of 22 questions; it took approximately $7 \mathrm{~min}$ for participants to respond. Reward points valued at 50 yen were provided as incentives for participation (approximately 0.5 US dollars as of June 2020). This study was approved by the Ethics Committee of Tokyo Medical University, Tokyo, Japan (No: T2019-0234). Informed consent was obtained from all respondents.

Assessment of the frequency of hand hygiene implementation in moments requiring hand hygiene: Among the moments where hand hygiene should be practiced, as recommended by the MHLW and CDC for ordinary citizens, this study assessed the frequency of hand hygiene implementation in 5 moments that were related to daily life practices for many people (after returning from a public place, after using the toilet, after touching something outside, before eating food, and after blowing your nose, coughing, or sneezing) $(8,9)$. In each of these 5 moments, participants were asked about the frequency of implementation of hand hygiene during the previous week and responded using a 4-point-Likert scale (1: "Always", 2: "Sometimes", 3: "Rarely", or 4: "Never").

Assessment of the total number of hand hygiene practices per day: Participants reported the average number of handwashing sessions using soap and water per day and the average number of times alcohol-based hand sanitizers were used per day. The sum of the 2 was defined as the total number of hand hygiene practices per day.

Assessment of sociodemographic factors: In the first wave survey, participants stated their sex, age, smoking status (smokers/non-smokers), underlying diseases including heart diseases, respiratory diseases, kidney diseases, diabetes, and hypertension (yes/ no), marital status (not married/married), working status (working/not working), and residential area (metropolitan area [i.e., Tokyo, Kanagawa, Saitama, and Chiba]/non-metropolitan areas [i.e., Ibaraki, Tochigi, and Gunma]). The research company also provided categorized data for the following parameters: living arrangement (with others/alone), educational attainment (university graduate level or above), and household income level $(<5$ million yen or $\geq 5$ million yen per year).

Statistical analysis: Regarding the status of implementation in the 5 moments related to daily life practices, when a participant responded with "1" ("Always") on the 4-point-Likert scale, it was considered that hand hygiene was being performed in that specific moment. We clarified the percentage of individuals practicing hand hygiene at each moment and at all 5 moments.

Regarding the number of hand hygiene practices, the distribution of the total number of hand hygiene practices per day was initially described using histograms and boxplots. Subsequently, receiver operating characteristic (ROC) curve analysis was performed using the Youden Index (14), and the cutoff 
Hand Hygiene in Japan during the COVID-19 Pandemic

Table 1. Participants' characteristics

\begin{tabular}{lcc}
\hline & \multicolumn{2}{c}{$N=2,149(100 \%)$} \\
& $n$ & $\%$ \\
\hline Sex (men) & 1,096 & 51.0 \\
Age, years & $50.3^{1)}$ & $16.1^{2)}$ \\
Smoking (smoker) & 311 & 14.5 \\
Underlying diseases ${ }^{3)}$ (yes) & 549 & 25.5 \\
Marital status (married) & 1,225 & 57.0 \\
Working status (working) & 1,362 & 63.4 \\
Residential area (metropolitan area $\left.{ }^{4)}\right)$ & 1,945 & 90.5 \\
Living arrangement (with others) & 1,697 & 79.0 \\
Educational attainment (university graduate level or above) & 1,147 & 53.4 \\
Household income level ( $\geq 5$ million yen per year) & 1,118 & 52.0 \\
\hline 1): Mean. & & \\
2): Standard deviation. & & \\
3): Underlying diseases included heart diseases, respiratory diseases, kidney \\
$\quad$ diseases, diabetes, and hypertension. & & \\
4): Included Tokyo, Kanagawa, Saitama, and Chiba prefecture. & &
\end{tabular}

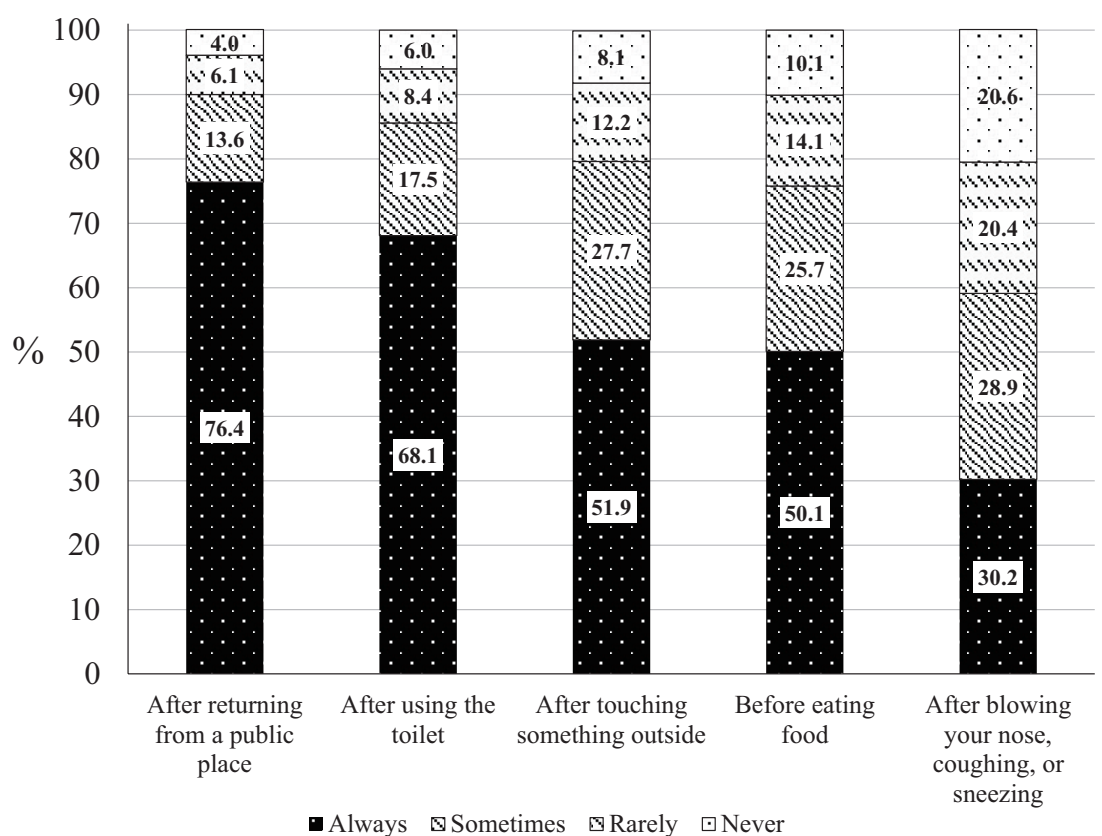

Fig. 1. Distribution of frequency of hand hygiene implementation in each moment. Regarding each of the 5 moments, the participants were asked about the frequency of implementing hand hygiene practices during the previous week and responded using a 4-point-Likert scale (1: "Always", 2: "Sometimes", 3: "Rarely", or 4: "Never"). In this study, when a participant responded 1 ("Always") on 4-point-Likert scale, it was considered that hand hygiene was performed in that specific moment.

value of the total number of hand hygiene practices per day, which determined whether hand hygiene was being practiced in all 5 moments, was clarified. The sensitivity, specificity, and area under the curve have been reported. Based on previous studies (15), the area under the curve values were interpreted as excellent $(\geq 0.90)$, good $(0.80-0.89)$, fair $(0.70-0.79)$, and poor $(<0.70)$.

$\mathrm{R}$ version 4.0.1 and "ROCR" from the R package (version 1.0-11)were used to perform all statistical analyses.

\section{RESULTS}

The internet research company reached out to 2,369 participants; after excluding participants who were not registered with the company at the time of the 4th wave survey $(n=31), 2,149$ participants responded to the questionnaire (Table 1).

Figure 1 shows the proportion of individuals practicing hand hygiene at each time point. The percentage of individuals practicing hand hygiene ranged from $30.2 \%$ to $76.4 \%$. In particular, the prevalence of implementing hand hygiene after touching something outside, before eating food, and after blowing the nose, coughing, or sneezing was $51.9 \%, 50.1 \%$, and $30.2 \%$, respectively. The prevalence of participants who did not perform any hand hygiene at any moment was $2.4 \%$. Last, only $21.1 \%$ of individuals practiced hand hygiene at all times. 


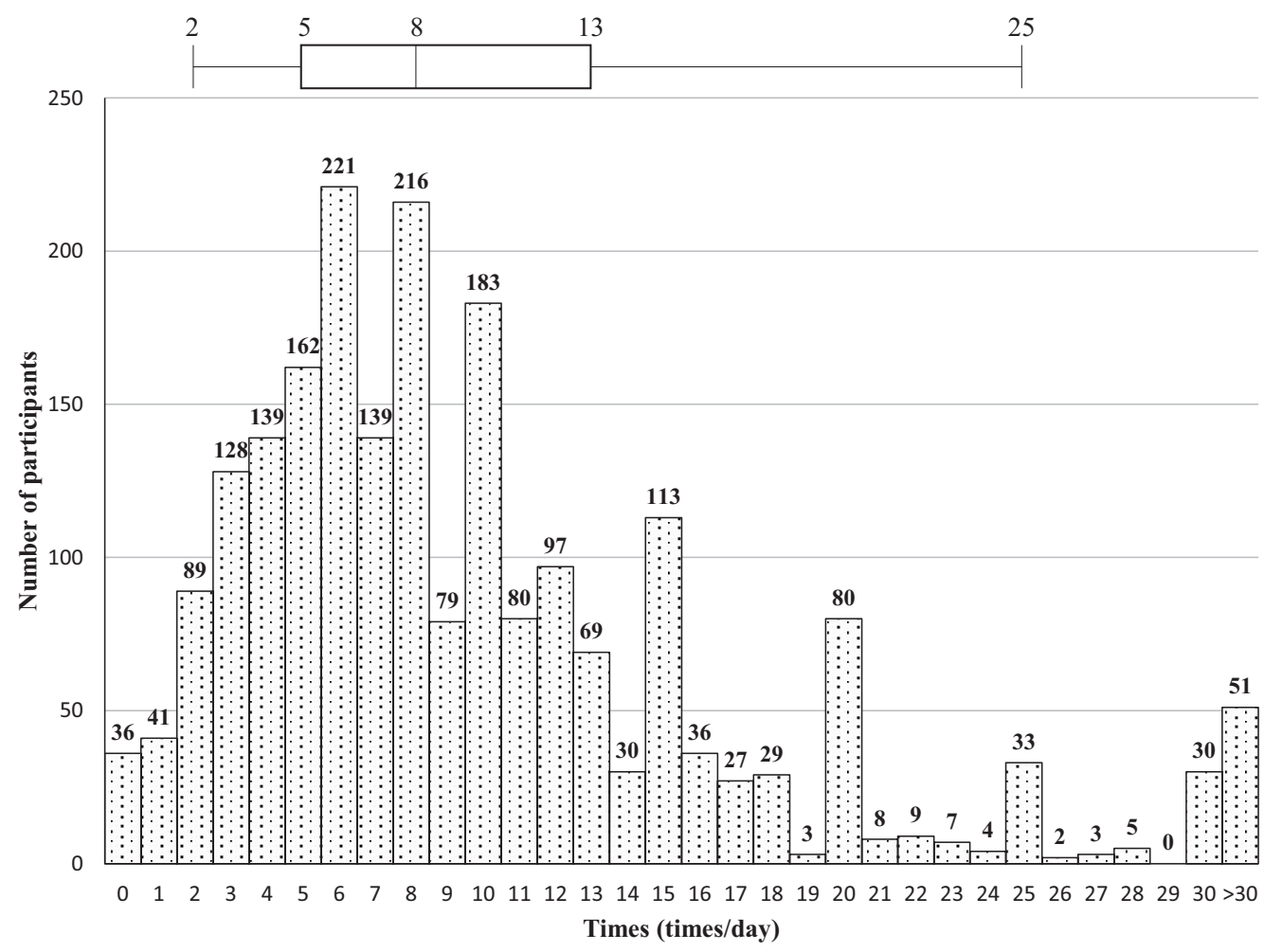

Fig. 2. Histogram and boxplot of the total number of hand hygiene practices per day.

Figure 2 shows a histogram and boxplot of the total number of hand hygiene practices per day. The mean, median, 25th percentile, and 75th percentile for handwashing using soap and water were 6.6 times/ day, 5 times/day, 3 times/day, and 10 times/day (range: 0-53), respectively; for hand hygiene, which included handwashing using soap and water and alcohol-based hand sanitizers, it was $10.2,8,5$, and 13 times/day, respectively (range: $0-110$ ).

Figure 3 and Table 2 show the results of the ROC curve analysis. When the cutoff value was 9 times/ day, the Youden Index reached the maximum (Youden Index, 0.372; sensitivity, 74.9\%; specificity, $62.4 \%$ ). Furthermore, when the cutoff values were 10 times/day or 11 times/day, although the Youden Index was similar to the cutoff value of 9 times/day, high specificity was obtained (10 times/day; Youden Index, 0.366; specificity, 65.9\% / 11 times/day; Youden Index, 0.365; specificity, $74.4 \%$ ).

\section{DISCUSSION}

We set out to clarify the status of hand hygiene implementation among ordinary citizens during the COVID-19 pandemic and calculated the standard value of the number of hand hygiene practices per day as an indicator of education and assessment. As for the implementation status of hand hygiene at each moment, only $21.1 \%$ of individuals practiced hand hygiene in all 5 moments related to daily life behavior. This study elucidates that there is still room for improvement in the implementation status of hand hygiene among ordinary citizens. Furthermore, ROC curve analysis revealed that the total number of hand hygiene practices per day was 9-11 times/day or more among individuals who were

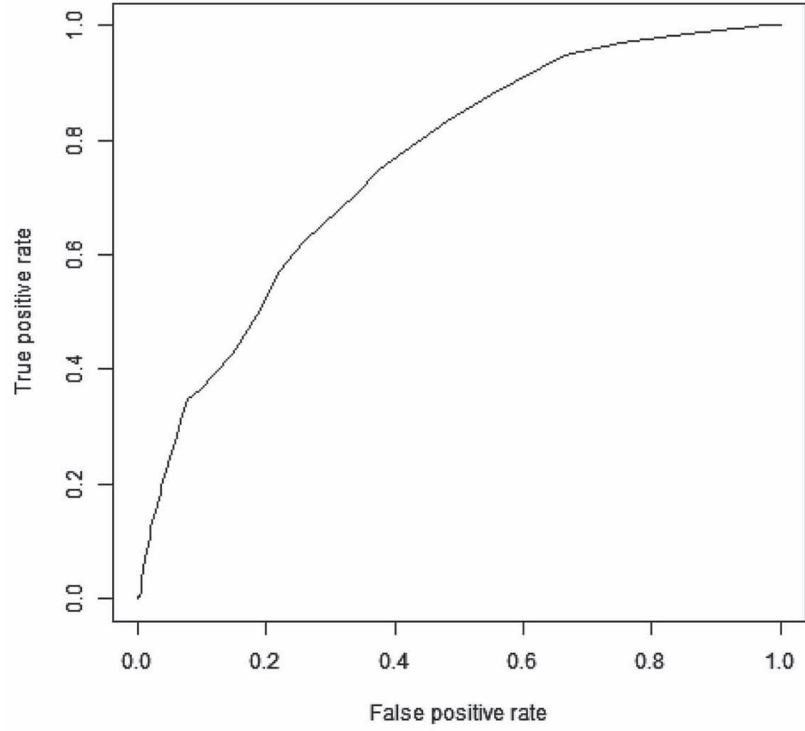

Fig. 3. Receiver operating characteristic (ROC) curve analysis for the cutoff value of the total number of hand hygiene practices per day that determines whether hand hygiene was practiced in all 5 moments. The 5 moments are: after returning from a public place; after using the toilet; touching an item or surface that may be frequently touched by other people; before eating food; and after blowing your nose, coughing, or sneezing. Area under the curve (AUC): 0.757 (95\% confidence interval: 0.733-0.780).

able to practice the measure in all 5 moments. However, the sensitivity and specificity were considerably different for these 3 frequencies. As a certain degree of specificity is essential for the implementation and education of more reliable preventive actions during a pandemic, it may be logical to set the standard value at 
Table 2. Cutoff values and coordinates and Youden Index of the receiver operating characteristic (ROC) curve analysis

\begin{tabular}{lccc}
\hline $\begin{array}{l}\text { Cutoff value } \\
\text { (total number of hand hygiene } \\
\text { practices per day [times/day]) }\end{array}$ & Sensitivity $(\%)$ & Specificity $(\%)$ & Youden Index \\
\hline 0 & 100.0 & 0.0 & 0.000 \\
1 & 100.0 & 2.1 & 0.021 \\
2 & 99.8 & 4.5 & 0.043 \\
3 & 99.3 & 9.6 & 0.090 \\
4 & 98.2 & 16.9 & 0.151 \\
5 & 96.9 & 24.7 & 0.216 \\
6 & 94.7 & 33.7 & 0.284 \\
7 & 87.9 & 44.9 & 0.328 \\
8 & 83.5 & 51.9 & 0.354 \\
9 & 74.9 & 62.4 & 0.372 \\
10 & 70.7 & 65.9 & 0.366 \\
11 & 62.1 & 74.4 & 0.365 \\
12 & 57.3 & 77.8 & 0.351 \\
13 & 49.6 & 81.5 & 0.310 \\
14 & 44.5 & 84.2 & 0.287 \\
15 & 42.7 & 85.5 & 0.282 \\
16 & 36.3 & 90.4 & 0.268 \\
17 & 34.8 & 92.2 & 0.270 \\
18 & 31.9 & 93.0 & 0.249 \\
19 & 28.6 & 93.8 & 0.224 \\
20 & 28.4 & 93.9 & 0.223 \\
\hline
\end{tabular}

11 times/day or more for this purpose.

The overall implementation rate of hand hygiene practices in moments requiring hand hygiene was as low as $30.4-76.4 \%$. In particular, the implementation was low in moments after touching something outside, before eating food, and after blowing your nose, coughing, or sneezing, and lowest after blowing the nose, coughing, or sneezing. As coughing and sneezing occur suddenly, it may be difficult to practice hand hygiene. Additionally, hand hygiene for this moment is mainly for mitigating the spread of infection to others while the other 4 moments are meant to protect oneself from infection. Therefore, ordinary citizens may not pay as much attention to this moment compared to the other 4 moments. The MHLW is already conducting educational activities for these moments through its website (8); however, implementation by citizens is still insufficient, and further educational interventions are required.

It is ideal for ordinary citizens to understand specific moments when hand hygiene should be followed and practiced. On the other hand, we also believe that the amount of information disseminated about these moments may be overwhelming for ordinary citizens, which could be counterproductive. With this in mind, we suppose that it is possible to convey a more comprehensible and precise informative message when conducting educational activities on hand hygiene, such as by indicating the exact number of hand hygiene practices necessary with the appropriate hand hygiene moments. Therefore, in this study, we used ROC curve analysis to calculate the standard number of hand hygiene practices per day as an indicator of education and assessment. The Youden Index was highest when the cutoff value was 9-11 times/day. Specificity is considered important from the viewpoint of implementing certain preventive actions and raising awareness. In other words, if the specificity is low, the false-positive rate becomes high, which could mean that persons are assessed as engaging in sufficient hand hygiene practices, even if they are not practiced at the appropriate time. From this perspective, the $62.4 \%$ specificity is insufficient if the number of events is 9 times/day. It may be more logical to set the cutoff value at 11 times/day or more, although it is debatable whether $74.4 \%$ specificity (cutoff value: 11 times/day) is sufficient. Additionally, a previous study involving a telephone interview survey in Hong Kong during the severe acute respiratory syndrome pandemic reported that practicing hand hygiene 11 times/day or more was a protective factor against the disease (16). The basis of this previous study and our study were selfreported evaluations; thus, the effect of recall bias was unavoidable (17). Additionally, it is possible that social desirability bias may have led to an overestimation of the implementation status (18). Nevertheless, obtaining self-reported responses about whether hand hygiene was performed 11 times or more may be an indicator that reflects the actual status of hand hygiene implementation. Further, upon focusing on the specificity of the results of the ROC curve analysis, when the cutoff value was 16 times/day, the Youden Index was as low as 0.268 , but specificity was $90 \%$ or more. If the pandemic worsens, which would require 
more rigorous education and assessment of preventive actions, it may be favorable to set the handwashing standard as 16 times or more.

There are some limitations that should be considered in our study. First, the most important point is that in this study, participants were recruited from people enrolled in a single internet research company; thus, the results may have been affected by selection bias. Relatively little is known about the characteristics of people in online communities (19). According to the latest White Paper 2019 issued by the Japanese government (20), the percentage of those who use the Internet regularly was approximately $95 \%$ for people in their 20 s to $50 \mathrm{~s}$, and $76.6 \%$ and $51.0 \%$ for those in their $60 \mathrm{~s}$ and 70 s, respectively. In addition, it has been reported that individuals who use the Internet regularly tend to have higher incomes than those of non-users (20). Therefore, it can be said that the participants in this study may be more educated or have a higher income than that of the average Japanese population. Additionally, when comparing the age and sex composition of participants in this study to the latest population estimates reported by the Japanese government (21), there were more people in their 20s and fewer in their 40s in this study. Considering these facts, the results of this study, such as percentages, should be interpreted with caution, as they may have been affected by selection bias. Second, in this study, the frequency and number of hand hygiene practices were assessed using a self-reported questionnaire. Because self-reported questionnaires are subject to the influence of recall bias and social desirability bias, the frequency and number of events revealed in this study may be overestimated $(17,18)$. However, despite this fact, only $21.1 \%$ of individuals responded that they practiced hand hygiene at all times. Finally, the results may not be applicable to other population groups. In the case of other populations with different cultural, ethnic, and geographical backgrounds, the frequency and number of hand hygiene practices may vary greatly when compared with those reported in the present survey. Despite these limitations, to the best of our knowledge, this is the first study to clarify the status of hand hygiene implementation of ordinary citizens during the COVID-19 pandemic and present a standard value of the number of hand hygiene practices per day, as an indicator of awareness and assessment.

In conclusion, only a limited number of individuals in Japan always practiced hand hygiene in significant moments during the COVID-19 pandemic, at a time when there is increased attention and awareness for preventive actions. Further educational activities on the implementation of hand hygiene are required. The criterion (total number of hand hygiene events: 11 times/day or more) may be beneficial for education and evaluation purposes.

Acknowledgments We express our sincere gratitude to all the participants who enrolled in this study. This study was supported by a grant from Meiji Yasuda Life Foundation of Health and Welfare.

Conflict of interest None to declare.

\section{REFERENCES}

1. Qualls N, Levitt A, Kanade N, et al. Community Mitigation Guidelines to Prevent Pandemic Influenza - United States, 2017. MMWR Recomm Rep. 2017;66:1-34.

2. Ferretti L, Wymant C, Kendall M, et al. Quantifying SARSCoV-2 transmission suggests epidemic control with digital contact tracing. Science. 2020;368:eabb6936.

3. Wiersinga WJ, Rhodes A, Cheng AC, et al. Pathophysiology, transmission, diagnosis, and treatment of coronavirus disease 2019 (COVID-19): a review. JAMA. 2020;324:782-93.

4. Leung CC, Lam TH, Cheng KK. Mass masking in the COVID-19 epidemic: people need guidance. Lancet. 2020;395:945.

5. Feng S, Shen C, Xia N, et al. Rational use of face masks in the COVID-19 pandemic. Lancet Respir Med. 2020;8:434-6.

6. World Health Organization (WHO). Coronavirus disease (COVID-19) advice for the public. Available at $<$ https://www. who.int/emergencies/diseases/novel-coronavirus-2019/advicefor-public $>$. Accessed July 16, 2020.

7. WHO. Recommendations to Member States to improve hand hygiene practices to help prevent the transmission of the COVID-19 virus. Available at $<$ https:/www.who.int/publications/ i/item/recommendations-to-member-states-to-improve-handhygiene-practices-to-help-prevent-the-transmission-of-the-covid19-virus $>$. Accessed July 16, 2020.

8. Japanese Ministry of Health, Labour and Welfare. Prevention for new coronavirus. Available at <https://www.mhlw.go.jp/ stf/seisakunitsuite/bunya/0000121431_00094.html\#yobou>. Accessed July 16, 2020. Japanese.

9. U.S. Centers for Disease Control and Prevention. When and How to Wash Your Hands. Available at $<$ https://www.cdc.gov/ handwashing/when-how-handwashing.html>. Accessed July 16, 2020 .

10. Machida M, Nakamura I, Saito R, et al. Adoption of personal protective measures by ordinary citizens during the COVID-19 outbreak in Japan. Int J Infect Dis. 2020;94:139-44.

11. Machida M, Nakamura I, Saito R, et al. Incorrect use of face masks during the current COVID-19 pandemic among the general public in Japan. Int J Environ Res Public Health. 2020;17:6484.

12. Japanese Ministry of Health, Labour and Welfare. Coronavirus disease (COVID-19) situation within the country. Available at $<$ https://www.mhlw.go.jp/stf/covid-19/kokunainohasseijoukyou. html $>$. Accessed October 2, 2020. Japanese.

13. The Prime Minister of Japan and His Cabinet. [COVID-19] Press conference by the Prime Minister regarding the novel coronavirus. Available at $<$ https://japan.kantei.go.jp/98 abe/ statement/202005/_00003.html>.Accessed October 2, 2020.

14. Youden WJ. Index for rating diagnostic tests. Cancer. 1950;3:325 .

15. Metz CE. Basic principles of ROC analysis. Semin Nucl Med. 1978;8:283-98.

16. Lau JTF, Tsui H, Lau M, et al. SARS transmission, risk factors, and prevention in Hong Kong. Emerg Infect Dis. 2004;10:58792.

17. Coughlin SS. Recall bias in epidemiologic studies. J Clin Epidemiol. 1990;43:87-91.

18. Paulhus DL. Two-component models of socially desirable responding. J Pers Soc Psychol. 1984;46:598-609.

19. Wright KB. Researching Internet-based populations: advantages and disadvantages of online survey research, online questionnaire authoring software packages, and web survey services. J Comput Mediat Commun. 2017;10.

20. Ministry of Internal Affairs and Communications, Japan. Information and Communications in Japan, WHITE PAPER 2019. Available at <https://www.soumu.go.jp/johotsusintokei/ whitepaper/eng/WP2019/2019-index.html > . Accessed October 2, 2020.

21. Statistics Bureau of Japan. Population Estimates by Age (FiveYear Groups) and Sex-February 1, 2020 (Final estimates), July 1, 2020 (Provisional estimates). Available at $<$ https://www.e-stat. go.jp/en $/$ stat-search/files?page $=1 \&$ layout $=$ datalist $\&$ toukei $=002$ $00524 \&$ tstat $=000000090001 \&$ cycle $=1 \&$ year $=20200 \&$ month $=$ $23070907 \&$ tclass $1=000001011678 \&$ result_back $=1>$. Accessed October 2, 2020. 